\title{
Simple and compact neutral metal vapor laser assembly operating in a low- temperature region
}

\author{
Hiroshi Saito, Hiroshi Taniguchi, and Hajime Nozawa ${ }^{\text {a) }}$ \\ Department of Electronic Engineering, Faculty of Engineering, Iwate University, Ueda 4-3-5, \\ Morioka-shi 020, Japan \\ Ken-ichi Owashi \\ Department of Electrical Engineering, Faculty of Engineering, Iwate University, Ueda 4-3-5, \\ Morioka-shi 020, Japan
}

(Received 22 July 1985; accepted for publication 4 September 1985)

\begin{abstract}
A compact and inexpensive technique is reported for a discharge-excited pulsed metal vapor laser (MVL) at a low operating temperature utilizing an air-blown-type spark-gap switch. A laser is excited in an aperiodic pulse train by successive pulsed discharges of a storage capacitor through a spark-gap switch. A variety of neutral metal vapor laser (MVL) using metal compound as a lasant is briefly reported with the compact device.
\end{abstract}

\section{INTRODUCTION}

An elemental metal vapor laser (MVL) possesses some excellent features ${ }^{1}$ such as high output, high efficiency, high gain, large cross section of the output beam, and high pulse repetition frequency; although a major disadvantage is its high operating temperature. Substitution of a metal halide, ${ }^{2-5}$ volatile metal compound, and/or organometallic compound $^{6-9}$ as the lasant permits operation in a low temperature and allows considerable simplification of the design of the laser tube. MVL will be used extensively because of the great variety and if it is simple and easy to handle. This paper describes a compact and inexpensive technique for the pulsed MVL system at a low operating temperature.

\section{APPARATUS}

\section{A. General description of the MVL system}

An electrical circuit used to excite the MVL is shown in Fig. 1, where a handmade air-blown-type spark-gap switch (GS) is used as a substitute for the usual thyratron. By using this GS, a compact and inexpensive MVL system can be realized.

A laser is driven by successive pulsed discharges of an energy storage capacitor $(C=4 \times 1 \mathrm{nF}$; ceramic capacitor of doorknob type) through the free-running GS. This is done in an aperiodic pulse train as a boosted-ac $(50 \mathrm{~Hz})$ high voltage

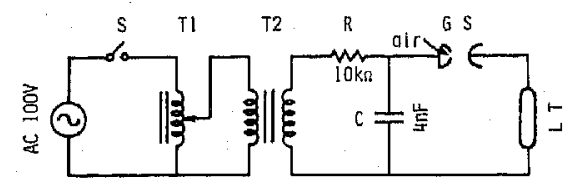

FIG. 1. An electrical circuit used to excite the metal vapor laser. $S$ : knife switch, T1:0-130 V, 2-kV A autotransformer, T2: 1:60,3-kV A single-phase transformer, GS: air-blown-type spark-gap switch, LT: laser tube, C: energy storage capacitor. of about $5.4 \mathrm{kV}$ rms, during each half-cycle of the $50-\mathrm{Hz}$ ac.

Flowing He gas is used as a buffer gas in order to purge the discharge products from the tube by a vacuum pump together with the gas flow. He gas flow rate and internal pressure $(p)$ of the tube are kept throughout experiments. Their values are in the region of $15-30 \mathrm{cc} / \mathrm{min}$ and $2-20$ Torr.

The optical resonator of $\sim 55-\mathrm{cm}$ spacing is formed by a flat dielectric-coated mirror with high reflectivity and an output coupler of a flat dielectric-coated mirror with an adequate transmittance at a desirable wavelength.

\section{B. GS construction}

Figure 2 shows a schematic construction of the GS. The electrodes of the GS are made of brass. An $\sim 2-\mathrm{mm}$ hole is drilled in the center of the one-sided electrode and compressed air $\left(1.5-2.0 \mathrm{~kg} \mathrm{~cm}^{-2}\right.$ gauge) is blown to the opposite electrode through the hole. The spacing between the electrodes is adjusted by screwing one of the rods in and out, thus the discharge voltage and the pulse repetition rate are controlled. Typical spacing of the GS electrodes $(d)$ in the present system is about $1 \mathrm{~mm}$ for applied voltage $\left(V_{a}\right)$ and about $5.4 \mathrm{kV}$ rms and about $10-\mathrm{kHz}$ repetition rate of pulsed discharges.

\section{Laser tube (LT) construction}

Figure 3 shows a construction of the laser tube (LT). A LT is made of a 1.2-1.3-cm-i.d. T-shaped Pyrex or quartz

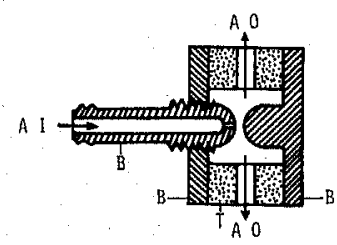

FIG. 2. A schematic construction of the air-blown-type spark-gap switch. AI compressed air in, $\mathrm{AO}$ : air out, $\mathrm{B}$ : brass, $\mathrm{T}$ : Teflon: 


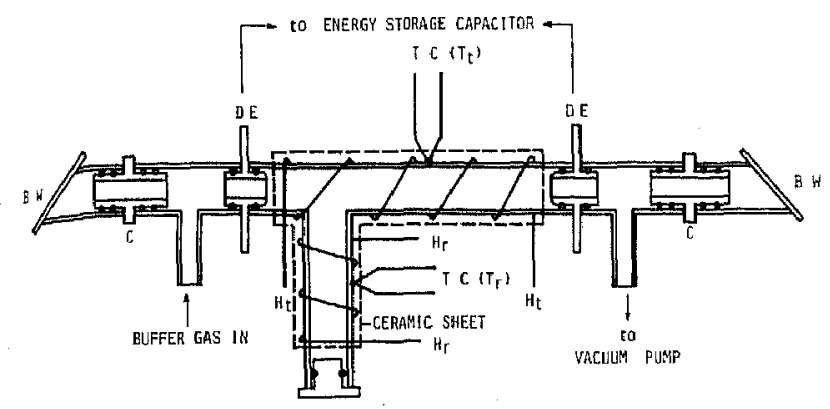

FIG. 3. Axial cross-sectional view of the metal vapor laser tube. BW: Brewster window, $\mathrm{C}$ : connector, DE: discharge electrode, $\mathrm{H}_{t}$ and $\mathrm{H}_{r}$ : heater for tube and reservoir heating, respectively, $\mathrm{TC}\left(T_{t}\right)$ and $\mathrm{TC}\left(T_{r}\right)$ : thermocouple for tube temperature $\left(T_{t}\right)$ and reservoir temperature $\left(T_{r}\right)$, respectively.

tube with Brewster windows made of photographic glass plate atboth ends. The annular discharge electrodes made of brass are separated by $17-20 \mathrm{~cm}$ and the total length of the laser tube is about $40 \mathrm{~cm}$. The i.d. of the discharge electrodes is about $0.5-0.6 \mathrm{~cm}$. The portion of the branch in the T-shaped tube is employed as a reservoir for the lasant. The metal vapor is introduced from the reservoir into the discharge region.

Outside the portions of the reservoir and the discharge tube, nichrome wire heaters are separately wound and wrapped in ceramic sheets for thermal shielding. As self-heating by repetitive pulsed discharges gradually raises the tube temperature, independent winding (of reservoir/tube winding) of the heaters is reasonable in order to obtain an adequate vapor pressure of volatile compounds and to control the vapor pressure for a practical use. The reservoir temperature $\left(T_{r}\right)$ and the discharge tube temperature $\left(T_{t}\right)$ in the center of each portion are measured independently by Chromel-Alumel thermocouples (TC). Rubber " $O$ " rings are used for gas sealing of the connections in the LT because of the low operating temperature. Thus quick assembly and disassembly of the LT become possible.

TABLE I. Metal compounds obtained from laser oscillation.

\begin{tabular}{lccc}
\hline \hline & $p$ & Oscillation & $\begin{array}{c}\text { Temperature } \\
\left({ }^{\circ} \mathrm{C}\right) \\
T_{t}\end{array}$ \\
\hline $\mathrm{Lasant} \mathrm{a}^{\mathrm{a}}$ & $\begin{array}{c}p \\
(\text { Torr })\end{array}$ & $T_{r}$ & $\simeq T_{r}$ \\
$\mathrm{CuCl}(\mathrm{AA})_{2}$ & $(5)$ & $300-600$, & $\simeq T_{r}$ \\
$\mathrm{Cu}(\mathrm{TAA})_{2}$ & $(2)$ & $140-160$, & $\simeq T_{r}$ \\
$\mathrm{Cu}(\mathrm{HFA})_{2}$ & $(3-7)$ & $94-120$, & $\simeq T_{r}$ \\
$\mathrm{MnCl}{ }_{2}$ & $(3)$ & $37-80$, & $\simeq T_{r}$ \\
$\mathrm{Mn}(\mathrm{AA})_{2}$ & $(5-20)$ & $600-750$, & $\simeq T_{r}$ \\
$\mathrm{Mn}(\mathrm{AA})_{3}$ & $(4)$ & $165-180$, & $\simeq T_{r}$ \\
$\mathrm{HAuCl} 4_{4} \cdot 4 \mathrm{H}_{2} \mathrm{O}^{\mathrm{b}}$ & $(4)$ & $120-130$ & $70-150^{\mathrm{c}}$ \\
\hline \hline
\end{tabular}

Detailed explanation was presented in Ref. 9.

bunpublished.

'With straight discharge tube, He flow rate was $110 \mathrm{cc} / \mathrm{min}$.
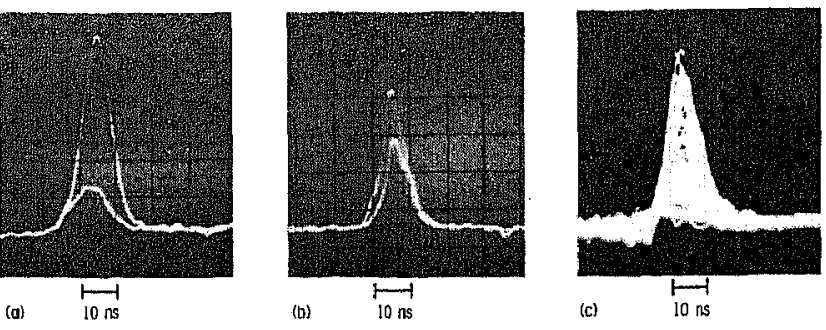

FIG. 4. Typical multiexposed oscilloscope traces of laser-output pulses. (a) $\mathrm{Cu}(\mathrm{AAA})_{2}, T_{r}=50^{\circ} \mathrm{C}, T_{t}=60^{\circ} \mathrm{C},(\mathrm{b}) \mathrm{Mn}(\mathrm{AAA})_{3}, T_{r}=180^{\circ} \mathrm{C}, T_{t}=185^{\circ} \mathrm{C}$ (c) $\mathrm{CuCl}: T_{r}=540^{\circ} \mathrm{C}, T_{1}=330^{\circ} \mathrm{C}$.

\section{EXPERIMENTAL RESULTS}

\section{A. GS operation and discharge characteristics}

A switching rate of the GS reached about $600 \mathrm{~Hz}$ and 10 $\mathrm{kHz}$, withoutand withblowing thecompressed air, respectively. It was under the conditions of optimum operation in the former, and of $d=1 \mathrm{~mm}, V_{a}=5.4 \mathrm{kV} \mathrm{rms}$ in the latter. The higher pulse repetition rate was achieved by removing the free ions between the GS electrodes with the compressed-air flow. Although an excitation pulse was aperiodic due to driving with an ac source, the time interval of each pulse in an aperiodic excitation pulse train was located in the range between the minimum and the maximum delay time $\mathrm{e}^{3}$ when a metal compound as a lasant was well dissociated and excited.

\section{B. Laser operation}

Laser operation was obtained using the metal halide of $\mathrm{CuCl}, \mathrm{MnCl}_{2}$, the metal $\beta$-diketone chelate of $\mathrm{Cu}(\mathrm{AA})_{2}$ (copper acetylacetonate), $\mathrm{Cu}(\mathrm{TAA})_{2}$ (copper trifluoroacetylacetonate), $\mathrm{Cu}(\mathrm{HFA})_{2}$ (copper hexafluoroacetylacetonate), $\mathrm{Mn}(\mathrm{AA})_{2}, \mathrm{Mn}(\mathrm{AA})_{3}$ (manganese acetylacetonate), and the volatile metal compound of $\mathrm{HAuCl}_{4}, 4 \mathrm{H}_{2} \mathrm{O}$ (chloroauric acid) as a lasant.

The laser-oscillation wavelength was 510.6 (and 578.2 $\mathrm{nm}$ at the higher temperature), 534.1 , and $627.8 \mathrm{~nm}$ for the copper, the manganese, and the gold compound, respectively. The metal compounds obtained from laser oscillation are listed in Table I. The lowest operating temperature of $37^{\circ} \mathrm{C}$ could be obtained using the $\mathrm{Cu}(\mathrm{HFA})_{2}$ chelate, which was prepared in our laboratory. Typical multiexposed oscilloscope traces of the laser output pulses are shown in Fig. 4. These figures indicate how the number of the oscillation pulses in the metal $\beta$-diketone chelate laser are few compared with that in the $\mathrm{CuCl}$ laser.

\section{DISCUSSION}

In summary, we were able to realize laser operation in a low-temperature region using a compact and inexpensive MVL system. Construction technique of the system was reported. 
The present system is not constructed to optimize the laser operation. The MVL operates only near the peak voltage of each half-cycle of the $50-\mathrm{Hz}$ ac because the driving voltage is low and the active length of the laser is relatively short compared with that of the usual device. Modification of the high-voltage power supply and the laser tube may lead to even more improved laser performance.

Further, this approach will enable us to use the MVL system using the other metal compounds.

\section{ACKNOWLEDGMENTS}

We would like to acknowledge the partial support by the Grant-in-Aid for Scientific Research from the Ministry of Education, Science, and Culture, and by the Hoso Bunka Foundation (HBF), in Japan. a) Present address: Tokyo Electronic Industry Co., Ltd., Asahigaoka 4-7-1, Hino-shi 191, Tokyo, Japan.

${ }^{1}$ W. T. Walter, N. Solimene, M. Piltch, and G. Gould, IEEE J. Quantum Electron. QE-2, 474 (1966).

${ }^{2}$ C. S. Liu, E. W. Sucov, and L. A. Weaver, Appl. Phys. Lett. 23, 92 (1973).

${ }^{3}$ C. J. Chen, N. M. Nerheim, and G. R. Russel, Appl. Phys. Lett. 23, 514 (1973).

${ }^{4}$ C. J. Chen, Appl. Phys. Lett. 24, 499 (1974).

${ }^{5}$ C. J. Chen, J. Appl, Phys. 45, 4663 (1974).

${ }^{6}$ A.J. Andrews, C. E. Webb, R. C. Tobin, and R. G. Denning, Opt. Commun. 22, 272 (1977).

${ }^{7}$ G. Chakrapani, T. A. P. Rao, A. A. N. Murty, and D. R. Rao, Appl. Phys. Lett. 31, 832 (1978).

${ }^{8}$ M. C. Gokay, M. Soltanolkotabi, and L. A. Cross, J. A.ppl. Phys. 49, 4357 (1978).

${ }^{9} \mathrm{H}$. Saito, H. Taniguchi, and T. Ishikawa, Tech. Rep. Iwate Univ. 18, 23 (1984). 